\title{
Comparison of the two most commonly used treatments for pyoderma gangrenosum: results of the STOP GAP randomised controlled trial
}

\author{
Anthony D Ormerod, ${ }^{1}$ Kim S Thomas, ${ }^{2}$ Fiona E Craig, ${ }^{3}$ Eleanor Mitchell, ${ }^{4}$ Nicola Greenlaw, ${ }^{5}$ \\ John Norrie, ${ }^{6}$ James M Mason, ${ }^{7}$ Shernaz Walton, ${ }^{8}$ Graham A Johnston, ${ }^{9}$ Hywel C Williams ${ }^{2}$ \\ on behalf of the UK Dermatology Clinical Trials Network's STOP GAP team
}

\section{ABSTRACT}

Division of Applied Medicine, Aberdeen University, Aberdeen, UK

${ }^{2}$ Centre of Evidence Based Dermatology, University of Nottingham, NG7 2NR, UK

3Department of Dermatology, Aberdeen Royal Infirmary,

Aberdeen, UK

${ }^{4}$ Nottingham Clinical Trials Unit, University of Nottingham, Nottingham, UK

${ }^{5}$ Robertson Centre for Biostatistics, University of

Glasgow, Glasgow, UK

${ }^{6}$ Centre for Healthcare

Randomised Trials, Aberdeen University, Aberdeen, UK

${ }^{7}$ School of Medicine, Pharmacy and Health, Durham University, Durham, UK

${ }^{8}$ Department of Dermatology, Hull Royal Infirmary, Hull, UK

${ }^{9}$ Department of Dermatology, Leicester Royal Infirmary,

Leicester, UK

Correspondence to:

K S Thomas

kim.thomas@nottingham.ac.uk Additional material is published online only. To view please visit the journal online (http://dx.doi. org/10.1136/bmj.h2958)

Cite this as: $B M J$ 2015;350:h2958 doi: 10.1136/bmj.h2958

Accepted: 27 April 2015

\section{OBJECTIVE}

\section{DESIGN}

SETTING prednisolone for the treatment of pyoderma gangrenosum, a painful, ulcerating skin disease with a poor evidence base for management.

Multicentre, parallel group, observer blind, randomised controlled trial.

39 UK hospitals, recruiting from June 2009 to November 2012.

\section{PARTICIPANTS}

121 patients ( 73 women, mean age 54 years) with clinician diagnosed pyoderma gangrenosum. Clinical diagnosis was revised in nine participants after randomisation, leaving 112 participants in the analysis set (59 ciclosporin; 53 prednisolone).

\section{INTERVENTION}

Oral prednisolone $0.75 \mathrm{mg} / \mathrm{kg} /$ day compared with ciclosporin $4 \mathrm{mg} / \mathrm{kg} /$ day, to a maximum dose of 75 and $400 \mathrm{mg} /$ day, respectively.

\section{MAIN OUTCOME MEASURES}

The primary outcome was speed of healing over six weeks, captured using digital images and assessed by blinded investigators. Secondary outcomes were time to healing, global treatment response, resolution of inflammation, self reported pain, quality of life, number of treatment failures, adverse reactions, and time to recurrence. Outcomes were assessed at
To determine whether ciclosporin is superior to

\section{WHAT IS ALREADY KNOWN ON THIS TOPIC}

Pyoderma gangrenosum is a severe, painful ulcerative skin condition that has a weak evidence base for treatments, with only one published randomised controlled trial of 30 participants

Prednisolone has been the main systemic treatment, but many clinicians now use ciclosporin in the belief that it is more effective and has fewer side effects

Both prednisolone and ciclosporin have important, predictable side effects

\section{WHAT THIS STUDY ADDS}

In this pragmatic randomised controlled trial, ciclosporin and prednisolone were of similar efficacy, but only $50 \%$ of ulcers had healed by six months,

Adverse events were common, occurring in around two thirds of participants taking either study drug suggesting that better treatments are required

The adverse event profile (serious infections with prednisolone, hypertension and renal dysfunction with ciclosporin) may help to inform decisions about treatment depending on underlying comorbidities baseline and six weeks and when the ulcer had healed (to a maximum of six months).

\section{RESULTS}

Of the 112 participants, 108 had complete primary outcome data at baseline and six weeks ( 57 ciclosporin; 51 prednisolone). Groups were balanced at baseline. The mean (SD) speed of healing at six weeks was $-0.21(1.00) \mathrm{cm}^{2} /$ day in the ciclosporin group compared with $-0.14(0.42) \mathrm{cm}^{2} /$ day in the prednisolone group. The adjusted mean difference showed no between group difference $\left(0.003 \mathrm{~cm}^{2} / \mathrm{day}\right.$, $95 \%$ confidence interval -0.20 to $0.21 ; \mathrm{P}=0.97$ ). By six months, ulcers had healed in 28/59 (47\%) participants in the ciclosporin group compared with $25 / 53$ (47\%) in the prednisolone group. In those with healed ulcers, eight (30\%) receiving ciclosporin and seven (28\%) receiving prednisolone had a recurrence. Adverse reactions were similar for the two groups $(68 \%$ ciclosporin and $66 \%$ prednisolone), but serious adverse reactions, especially infections, were more common in the prednisolone group.

\section{CONCLUSION}

Prednisolone and ciclosporin did not differ across a range of objective and patient reported outcomes. Treatment decisions for individual patients may be guided by the different side effect profiles of the two drugs and patient preference.

TRIAL REGISTRATION

Current Controlled Trials ISRCTN35898459.

\section{Introduction}

Pyoderma gangrenosum is a rare inflammatory disorder that causes progressive necrotising ulceration. A retrospective cohort study of UK cases reported an age and sex adjusted incidence of 0.63 per 100000 person years. ${ }^{1}$ Several variants of pyoderma gangrenosum have been recognised, but the classic form of the disease is the most commonly encountered. ${ }^{2}$ Manifestations of pyoderma gangrenosum are predominantly cutaneous, typically beginning as a tender erythematous nodule or pustule that rapidly breaks down to form a large, well demarcated ulcer with purplish, undermined edges. The condition is often observed in patients with an underlying systemic disease and has been particularly associated with inflammatory bowel disease, arthritis, and haematological malignancies. ${ }^{3}$ Approximately $25 \%$ of cases are precipitated by incidental or iatrogenic trauma, a phenomenon known as pathergy. ${ }^{4-7}$ The development of pyoderma gangrenosum is associated with a threefold increased risk of 
death (hazard ratio 3.03) compared with that of controls from the general population, and a $72 \%$ increased mortality over controls with inflammatory bowel disease. ${ }^{1}$

Currently there are no national or international guidelines covering the management of pyoderma gangrenosum. Patient information issued by the British Association of Dermatologists describes topical and systemic treatment options, as well as lesser used options such as intravenous steroids or biologics. ${ }^{8}$ The most commonly prescribed topical treatments for pyoderma gangrenosum are potent steroid preparations and calcineurin inhibitors, and commonly prescribed systemic treatments include steroids and immunosuppressants. $^{8}$

Only one randomised controlled trial in patients with pyoderma gangrenosum is reported in the literature. ${ }^{9}$ This small, placebo controlled study of 30 patients assessed infliximab, which is not considered to be a first line treatment for pyoderma gangrenosum. There is a complete lack of studies assessing the efficacy of commonly used treatments for pyoderma gangrenosum, so systematic reviews have primarily relied on anecdotal reports or retrospective case series. ${ }^{10}$ Given the complete absence of high quality evidence on treatments, we carried out a randomised controlled trial (STOP GAP, Study of Treatments fOr Pyoderma GAngrenosum Patients) to test the hypothesis that ciclosporin is superior to prednisolone in the treatment of pyoderma gangrenosum.

\section{Methods}

The trial protocol has been published previously. ${ }^{11}$

\section{Trial design and oversight}

We carried out a multicentre, parallel group, observer blind randomised controlled trial to compare the efficacy and safety of ciclosporin with that of prednisolone. Participants gave written informed consent. Oversight of the trial included a trial management group and independent trial steering and data monitoring committees. Patients suitable for topical treatment were entered into a parallel observational study, the results of which will be reported separately.

\section{Patient involvement}

Patients were involved in the design and conduct of this research. During the feasibility stage, priority of the research question, choice of outcome measures, and methods of recruitment were informed by discussions with patients through a focus group session and two structured interviews. During the trial, a patient joined the independent trial steering committee. Members of the UK Dermatology Clinical Trials Network also identified this research as being a priority area for clinicians treating patients with pyoderma gangrenosum. Once the trial has been published, participants will be informed of the results through a dedicated website (www.stopgaptrial.co.uk) and will be sent details of the results in a study newsletter suitable for a nonspecialist audience.

\section{Participants}

Recruitment took place at 39 hospitals in the United Kingdom. Participants were aged 18 years or more, with a diagnosis of pyoderma gangrenosum made by a recruiting dermatologist. Histopathology is rarely pathognomonic for pyoderma gangrenosum, and many clinicians avoid biopsy because of the risk of an immunological reaction that results in ulcer extension at the biopsy site. If the clinical diagnosis was uncertain, a biopsy was performed to exclude other diagnoses such as malignancy, granulomatous pyoderma gangrenosum, and arteritis, and advice was sought from an expert panel as necessary.

We excluded patients with pustular or granulomatous variants of pyoderma gangrenosum (as they may respond differently to treatment, and measurement of a single ulcer was not possible); patients receiving oral prednisolone, ciclosporin, or intravenous immunoglobulin in the previous month; patients participating in another clinical trial; women who were pregnant, lactating, or at risk of pregnancy; patients with known hypersensitivity to either of the study treatments; patients with clinically important renal impairment or other pretreatment findings that would result in the investigator not using either of the study drugs; patients with malignant or premalignant disease; patients with a concurrent medical condition for which treatments might interfere with ongoing treatment or cause harm; and patients taking rosuvastatin or those who had received a live vaccine in the two weeks before randomisation.

\section{Interventions}

Participants received oral prednisolone (brand chosen according to local practice) $0.75 \mathrm{mg} / \mathrm{kg} /$ day in a single dose or ciclosporin (Neoral; Novartis) $4 \mathrm{mg} / \mathrm{kg} /$ day in two divided doses. As this was a pragmatic trial, the dose could be adjusted according to normal practice, to a maximum of $1 \mathrm{mg} / \mathrm{kg} /$ day for prednisolone and $5 \mathrm{mg} /$ $\mathrm{kg} /$ day for ciclosporin. ${ }^{710}$ Topical treatment was prohibited during the trial. A change to the protocol was made in August 2011 (after 82 participants had been enrolled) as bowel perforation was experienced by a participant receiving prednisolone $110 \mathrm{mg} /$ day. As a result we implemented ceiling doses of $75 \mathrm{mg} /$ day for prednisolone and $400 \mathrm{mg} /$ day for ciclosporin.

\section{Randomisation and blinding}

Participants were randomised (1:1) to treatment allocation using a web based randomisation system hosted by Nottingham Clinical Trials Unit, using a computer generated pseudorandom list, with permuted blocks of randomly varying size between two and six (RALLOC add-on ${ }^{12}$ for Stata, TX). Randomisation was stratified by target lesion size $\left(<20 \mathrm{~cm}^{2} ; \geq 20 \mathrm{~cm}^{2}\right)$ and presence or absence of underlying systemic disease. It was not possible to blind clinicians and participants to treatment allocation owing to resource limitations and the complexities of different dosing regimens and safety testing of the two drugs. As a result, clinicians and participants were informed of their treatment allocation once data had been irrevocably entered into the randomisation 
database. Treatment allocation was concealed from the statistician and blinded assessors of the digital images until interventions were all assigned and recruitment, data collection, data cleaning, and blind analysis were complete.

Assessors blind to the allocated treatment assessed the ulcer size and global treatment response from digital images of the target lesion. If digital images were not available, the assessors used physical measurements of the lesion taken during clinic visits and global response by the treating clinician.

\section{Assessments}

Clinic visits took place at baseline, week 2, and week 6 (primary outcome) and when the ulcer had healed (up to a maximum of six months). Patient reported outcomes were collected from daily diaries or postal questionnaires. For participants whose pyoderma gangrenosum had healed, we assessed recurrence and time to recurrence from medical notes.

\section{Digital image assessments}

A template was photographed alongside the target ulcer to calibrate the image in the image analysis software (see supplementary figure). Two trained assessors mapped the circumference of the lesion using VERG Videometry VEV MD software (Vista Medical, Winnipeg, Canada). Two dermatologists independently reviewed all images to ensure that the lesions were consistent with a diagnosis of pyoderma gangrenosum and that the measurements taken by the trained assessors were an accurate representation of the ulcer's size.

\section{Outcomes}

\section{Primary outcome}

The main outcome measure was speed of healing over six weeks, captured for a single target lesion for each patient. If multiple lesions were present, we designated the largest lesion that could be photographed on a single plane as the target lesion.

We chose speed of healing for the primary outcome as previous studies have shown it to be a good predictor of healing in patients with leg ulcers, ${ }^{1314}$ and because blinded outcome assessment was possible using digital images and independent assessors. Assessing the primary outcome at six weeks also minimised loss to follow-up and the impact of participants switching to alternative treatments before primary outcome assessment. In cases where digital images were unavailable, or of poor quality, we used physical measurements of the ulcer taken by non-blinded clinicians at baseline and six weeks.

\section{Secondary outcomes}

Time to healing-defined as the time at which sterile dressings were no longer required (reported by patients and confirmed by clinicians at subsequent clinic visits). We identified this outcome as the most important of the secondary outcomes.

Pyoderma gangrenosum specific global treatment response-to assess treatment response we used a seven point Likert scale ranging from completely clear through to worse (assessed by clinicians and participants and from digital images for blinded assessment).

Resolution of inflammation-this was recorded by clinicians and participants using a scale reported by Foss. ${ }^{15}$

Self reported pain-participants self reported the severity of pain daily using a score from 0 to 4 (none, mild, moderate, severe, or extreme).

Health related quality of life-assessed using the dermatology life quality index ${ }^{16}$ and European quality of life-5 dimensions, three levels (EQ-5D-3L and EQ VAS). ${ }^{17} 18$

Time to recurrence-defined as the interval between the target lesion healing and a further episode of pyoderma gangrenosum (at any site). The period of follow-up available varied depending on the time at which participants were randomised into the trial.

Number of treatment failures-defined as those who withdrew from their randomised treatment because of treatment intolerance, whose pyoderma gangrenosum worsened, or whose target lesion remained unhealed after six months of follow-up.

Adverse reactions to study drugs-adverse events that were possibly, probably, or definitely related to the study drug.

\section{Sample size}

This was a superiority trial, with prednisolone as the control intervention. To provide $80 \%$ power (5\% level of significance) to detect a difference in means of 0.5 standard deviations in the primary outcome of speed of healing over six weeks, the target sample size was 140 participants, assuming a loss to follow-up of $10 \%$. We chose a difference of 0.5 standard deviations as being a clinically meaningful between group difference, as observational data suggested that ciclosporin was potentially more effective, but at higher cost, than prednisolone. ${ }^{19-21}$ As a result, we felt that a substantial treatment effect was necessary to justify a change in clinical practice.

\section{Statistical analysis}

We prespecified all analyses in a statistical analysis plan. Analysis was conducted using intention to treat principles; defined as all randomised participants, excluding those with a later diagnosis determined to be something other than pyoderma gangrenosum. We included all participants with available data at both the baseline and the six week visit in the primary analysis. The impact of missing values was explored in sensitivity analysis. A linear regression model was used to analyse differences between treatment groups for the primary outcome at six weeks, adjusting for the stratification variables.

Secondary outcomes were analysed using Cox regression models (for time to event outcomes); linear regression models for dermatology life quality index, EQ-5D, and EQ-VAS (adjusted for baseline values), and for self reported pain (which were summarised using area under the curve); proportional odds models for ordered 
categorical outcomes; and logistic regression models for binary outcomes. All analyses were adjusted for the stratification variables.

Sensitivity analysis of the primary outcome and time to healing were further adjusted for additional baseline variables including age, sex, weight, size of recruiting centre and geographical region; missing data; and participants who switched randomised treatments or received the trial drugs in combination during the period of the trial.

We analysed adverse reactions that occurred during the trial according to the original randomised allocation, regardless of whether other drugs had been introduced before the adverse reaction.

All statistical analyses were conducted using SAS software, version 9.2 and $R$ version 2.10.1.

\section{Results}

Of 499 patients screened from June 2009 to November 2012, 121 were eligible and gave written informed consent ( $86 \%$ of target of 140 participants) (fig 1).

Table 1 summarises the baseline characteristics of the participants. Nine were excluded after randomisation because histological findings failed to support a diagnosis of pyoderma gangrenosum. Such participants were randomised before confirmation of biopsy results as it was considered unethical to delay treatment for those with painful and rapidly spreading ulcers. As such, the intention to treat population was 112 participants (59 ciclosporin; 53 prednisolone). Baseline characteristics were balanced between the groups

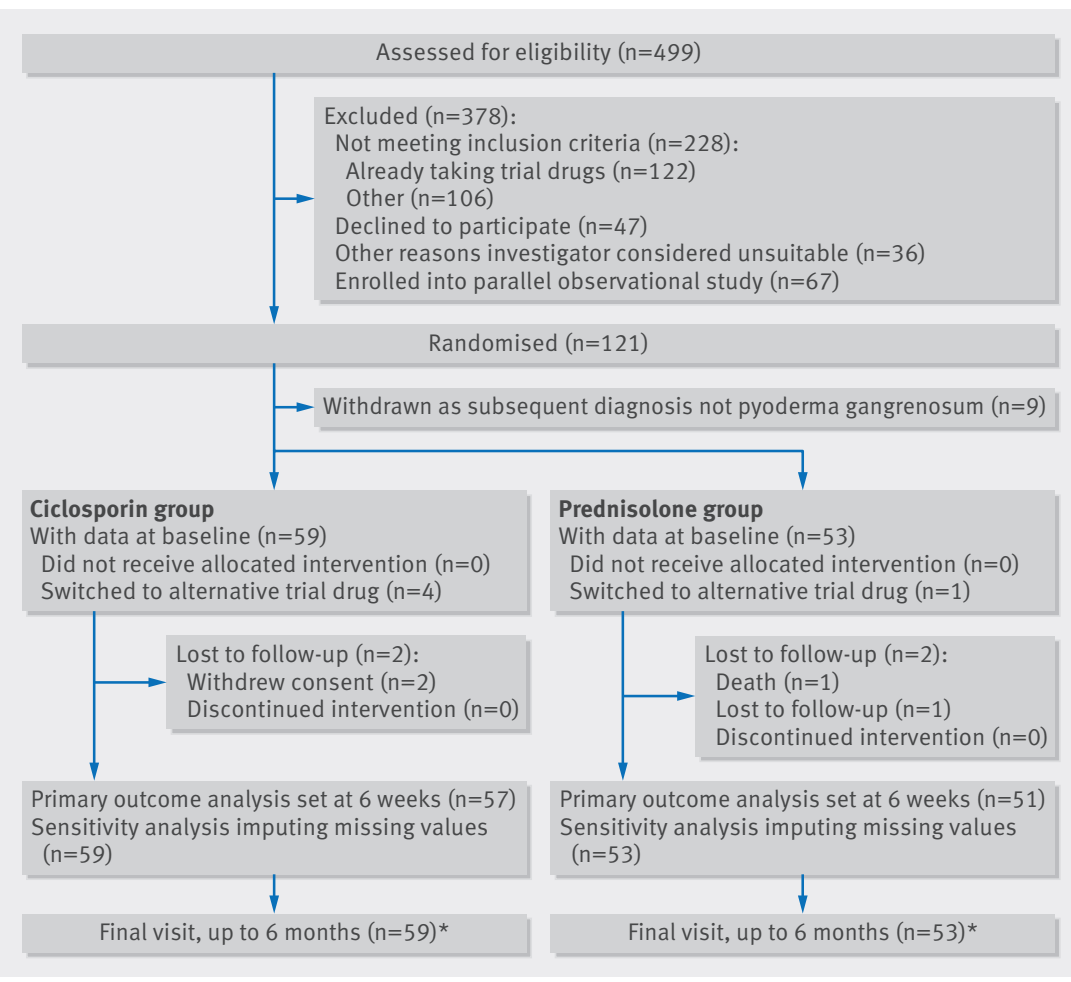

Fig 1| Flow of participants through study. *Number of patients with information on healing of lesion at any point up to six months after randomisation (main secondary outcome of time to healing) (table 1). Thirteen of the participants had previously been enrolled in the observational study of topical treatments but had failed to respond to treatment and so were subsequently re-consented for the randomised controlled trial.

During the trial, 16/112 (14\%) participants switched to the alternative trial drug and a further eight (7\%) received the two drugs together. Change in treatment occurred before the six week primary outcome assessment in five participants (prednisolone $\mathrm{n}=1$, ciclosporin $\mathrm{n}=4)$.

Nine participants $(8 \%)$ increased their dose of randomised drug during the trial: four in the prednisolone group, with increases ranging from $0.067 \mathrm{mg} / \mathrm{kg}$ daily to $0.6 \mathrm{mg} / \mathrm{kg}$ daily; and five in the ciclosporin group, with increases ranging from $0.36 \mathrm{mg} / \mathrm{kg}$ daily to $0.98 \mathrm{mg} / \mathrm{kg}$ daily.

Data on adherence to study drugs from daily diaries were available for 68/112 (61\%) participants. Of these, $36 / 37$ (97\%) in the ciclosporin group and 29/31 (94\%) in the prednisolone group took their treatment every day throughout the first six weeks of the trial.

\section{Primary outcome}

Of the 108 participants with data at baseline and six weeks, 86 (80\%) had blinded outcome data based on digital images. For the other $22(20 \%)$ participants whose digital images were either unavailable or of insufficient quality to allow assessment, healing speed was assessed using unblinded physical measurements taken during clinic visits.

There was no between group difference in speed of healing over six weeks (adjusted mean difference 0.003 $\mathrm{cm}^{2} /$ day, $95 \%$ confidence interval -0.20 to $0.21 ; \mathrm{P}=0.97$ ) (table 2). Similar results were observed for sensitivity analyses in which missing data were imputed (0.001 $\mathrm{cm}^{2} /$ day, -0.20 to $0.21 ; \mathrm{P}=0.99$ ), and separately, after adjusting for additional baseline covariates $\left(-0.10 \mathrm{~cm}^{2} /\right.$ day, -0.33 to 0.13 ; $\mathrm{P}=0.38$ ). Excluding the five patients who either swapped to the alternative trial drug or used both drugs in combination before the six week visit, did not change the overall treatment effect $(-0.036,-0.21$ to $0.14 ; \mathrm{P}=0.68$ ).

Additional post hoc analysis of data at week 2 showed no differences between the groups in onset of treatment response $(\mathrm{P}=0.21)$. For 33 participants (14 in the prednisolone group; 19 in the ciclosporin group) the pyoderma gangrenosum worsened (increased in size) between baseline and week 2 . The increases varied between patients, with a median increase in those that increased of $2.16 \mathrm{~cm}^{2}$ in the ciclosporin group and 2.55 $\mathrm{cm}^{2}$ in the prednisolone group.

For 64 participants (32 in each group) the pyoderma gangrenosum improved (decreased in size), with a median decrease of those that decreased of $1.96 \mathrm{~cm}^{2}$ in the ciclosporin group and $3.04 \mathrm{~cm}^{2}$ in the prednisolone group.

Nine participants (four in the prednisolone group; five in the ciclosporin group) showed no change in lesion area two weeks after baseline, and two had missing data at week 2 . 


\section{Secondary outcomes \\ Time to healing}

At six weeks, ulcers had healed in nine (15\%) participants in the ciclosporin group and $11(21 \%)$ in the prednisolone group. By six months, ulcers had healed in $28 / 59(47 \%)$ participants in the ciclosporin group and 25/53 (47\%) in the prednisolone group.

The Cox regression model for time to healing showed no significant difference between the interventions

\begin{tabular}{|c|c|c|}
\hline Characteristics & Ciclosporin $(n=59)$ & Prednisolone $(n=53)$ \\
\hline Mean (SD) age (years) & $57.2(16.9)$ & $51.3(15.2)$ \\
\hline Women & $42(71)$ & $31(58)$ \\
\hline White ethnicity & $55(93)$ & $53(100)$ \\
\hline Mean (SD) weight (kg), (range) & 88.4 (24.5), (50.0-171.0) & $93.2(27.2),(50.6-151.0)$ \\
\hline \multicolumn{3}{|l|}{ Underlying comorbidities: } \\
\hline Crohn's disease & $5(8)$ & $3(6)$ \\
\hline Ulcerative colitis & $7(12)$ & $8(15)$ \\
\hline Rheumatoid arthritis & $4(7)$ & $4(7)$ \\
\hline Other inflammatory arthritis & $3(5)$ & $3(6)$ \\
\hline Monoclonal gammopathy & $0(0)$ & $0(0)$ \\
\hline Myeloma & $0(0)$ & $0(0)$ \\
\hline Other malignancy & $4(7)$ & $0(0)$ \\
\hline Diabetes & $4(7)$ & $9(17)$ \\
\hline Mild renal impairment & $2(3)$ & $0(0)$ \\
\hline Epilepsy & $0(0)$ & $1(2)$ \\
\hline \multicolumn{3}{|l|}{ Characteristics of pyoderma gangrenosum } \\
\hline \multicolumn{3}{|l|}{ Type of pyoderma gangrenosum: } \\
\hline Classic & $50(85)$ & $47(89)$ \\
\hline Cribriform & $4(7)$ & $2(4)$ \\
\hline Peristomal & $2(3)$ & $2(4)$ \\
\hline Bullous & $0(0)$ & $1(2)$ \\
\hline Unsure & $3(5)$ & $1(2)$ \\
\hline Previous episode of pyoderma gangrenosum & $17(28)$ & $14(26)$ \\
\hline $\begin{array}{l}\text { Median area of target lesion }\left(\mathrm{cm}^{2}\right), \\
\text { (interquartile range) }\end{array}$ & $9.1,(3.6-24.7)$ & $8.1,(2.4-20.2)$ \\
\hline \multicolumn{3}{|l|}{ Location of lesion: } \\
\hline Arm & $2(3)$ & $1(2)$ \\
\hline Leg & $41(69)$ & $34(64)$ \\
\hline Other & $16(27)$ & $18(34)$ \\
\hline Mean (SD) No of lesions, (range) & $2.2(1.8),(1-10)$ & $2.6(2.4),{ }^{*}(1-12)$ \\
\hline
\end{tabular}

\begin{tabular}{|c|c|c|c|c|c|}
\hline $\begin{array}{l}\text { Treatment } \\
\text { group }\end{array}$ & $\begin{array}{l}\text { No in } \\
\text { group }\end{array}$ & $\begin{array}{l}\text { Mean (SD) speed of } \\
\text { healing }\left(\mathrm{cm}^{2} / \text { day }\right)\end{array}$ & $\begin{array}{l}\text { Mean difference } \\
\text { (ciclosporin- } \\
\text { prednisolone) }\end{array}$ & $\begin{array}{l}\text { Adjusted mean } \\
\text { difference* }(95 \% \mathrm{Cl})\end{array}$ & Pvalue \\
\hline Ciclosporin & 57 & $-0.21(1.00)$ & -0.074 & $0.003(-0.20$ to 0.21$)$ & 0.97 \\
\hline
\end{tabular}

*Adjusted for stratification variables lesion size and presence of underlying disease. Imputation of missing values ( $n=4)$ did not alter this result (see main text). (hazard ratio $0.94,95 \%$ confidence interval 0.55 to 1.63 ; $\mathrm{P}=0.84$ ) (table 3 and fig 2). Sensitivity analyses adjusting for additional baseline covariates was consistent with the main result (hazard ratio 1.01, 95\% confidence interval 0.57 to 1.79 ; $\mathrm{P}=0.98$ ), as was censoring the 16 participants who changed their treatment (hazard ratio $0.86,95 \%$ confidence interval 0.49 to $1.52 ; \mathrm{P}=0.60$ ).

Secondary outcomes did not differ significantly between the treatment groups, including global assessments of efficacy (fig 3), resolution of inflammation (tables 4 and 5), self reported pain in the first six weeks, quality of life over the duration of the study (table 6), health related quality of life (table 6), and time to recurrence (table 3). Treatment failure was documented in 29/59 (49\%) participants in the ciclosporin group and $26 / 53(49 \%)$ in the prednisolone group $(\mathrm{P}=0.88)$.

\section{Adverse reactions}

Overall, 40 (68\%) participants in the ciclosporin group and $35(66 \%)$ in the prednisolone group experienced at least one adverse reaction. Table 7 presents the specific events that occurred in at least 3\% of patients in either treatment group.

Adverse reactions differed between the treatments in line with known side effects of each drug. Notable differences included new onset diabetes and hyperglycaemia in the prednisolone group, and headaches, gastrointestinal disturbance, and renal dysfunction commonly in the ciclosporin group.

Nine serious adverse reactions occurred: two in the ciclosporin group (ruptured abdominal aortic aneurysm and acute kidney injury with increased serum creatinine $(212 \mu \mathrm{mol} / \mathrm{L})$ levels $)$ and seven in the prednisolone group (a bowel perforation; five serious infections (one resulted in death) that required admis-

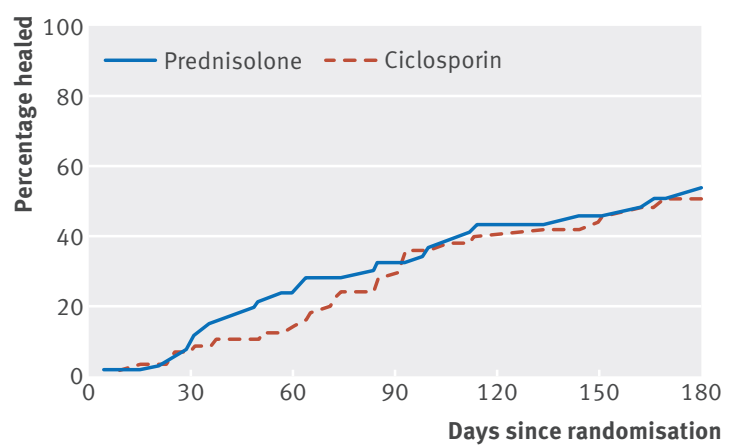

Fig 2 | Kaplan Meier plot showing time to healing of ulcer by treatment group

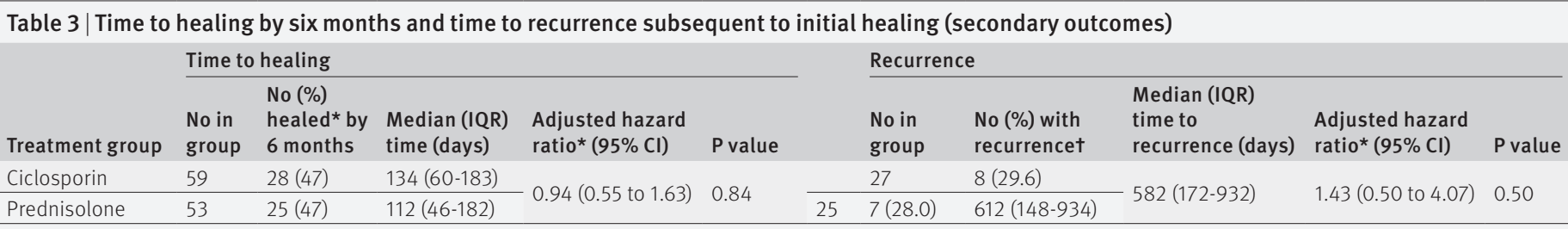



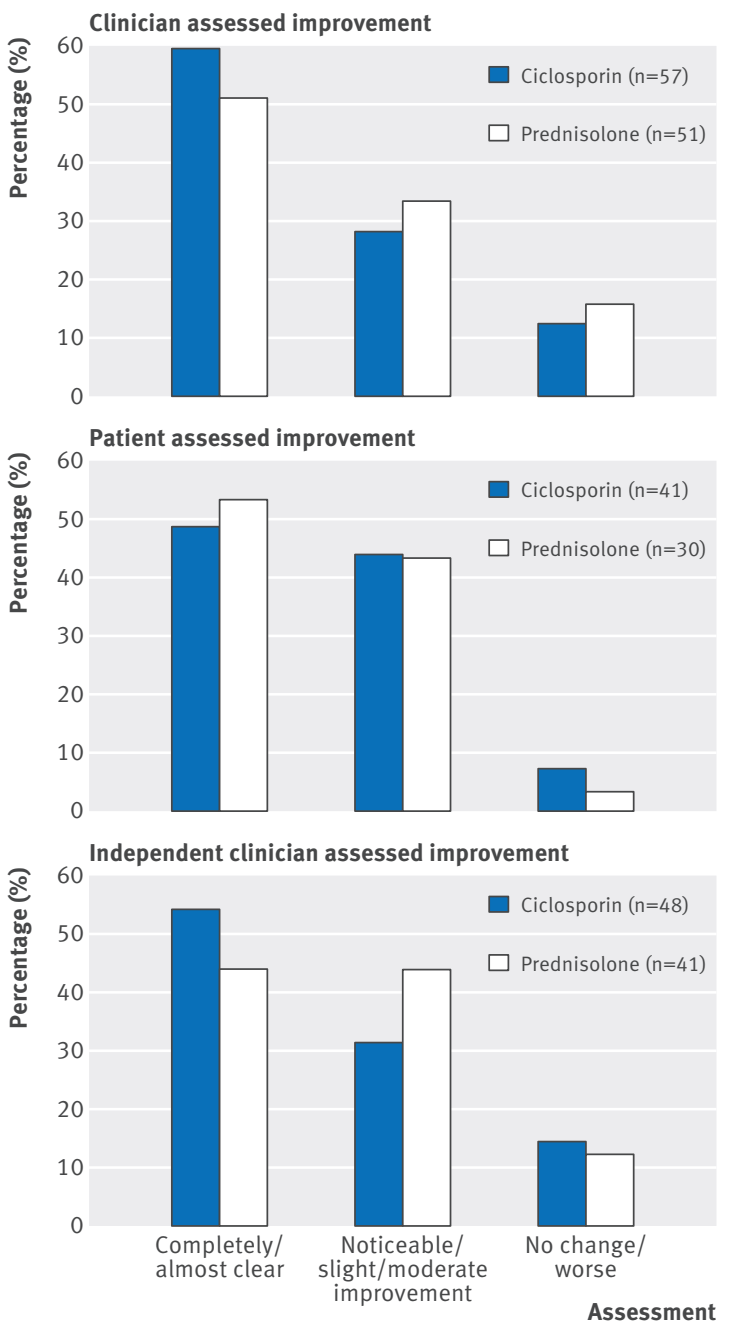

Fig 3 Global treatment response by clinician, patient, and independent clinician from digital images

sion to hospital or parenteral antibiotics; and one other infection).

\section{Discussion}

In this pragmatic randomised controlled trial, no difference was found between the two most commonly used treatments for pyoderma gangrenosum, ciclosporin and prednisolone, across a range of outcome measures. Contrary to the anecdotally derived belief that these drugs are efficacious in pyoderma gangrenosum, we found

\begin{tabular}{|c|c|c|c|c|}
\hline Treatment group* & No in group & $\begin{array}{l}\text { No (\%) with } \\
\text { resolution }\end{array}$ & Odds ratiot $(95 \% \mathrm{Cl})$ & P value \\
\hline \multicolumn{5}{|l|}{ Week 6: } \\
\hline Ciclosporin & 56 & $5(9)$ & \multirow{2}{*}{-1.03 (0.27 to 3.97) } & \multirow{2}{*}{0.96} \\
\hline Prednisolone & 51 & $6(12)$ & & \\
\hline \multicolumn{5}{|l|}{ Final visitł: } \\
\hline Ciclosporin & 57 & $10(18)$ & \multirow{2}{*}{1.11 (0.39 to 3.12) } & \multirow{2}{*}{0.85} \\
\hline Prednisolone & 51 & $10(20)$ & & \\
\hline
\end{tabular}

that fewer than half of the ulcers healed, even after prolonged treatment, and the speed of onset of response did not differ between the two treatments. Approximately two thirds of participants reported adverse reactions; $12 \%$ of whom experienced a serious event. The side effects observed were in line with the known side effect profiles of these drugs. More serious adverse reactions, especially serious infections, were reported in the prednisolone group, although patients receiving ciclosporin were at increased risk of renal toxicity.

A recent expert consensus paper considering safety, efficacy, and cost placed prednisolone as preferred treatment and ciclosporin as second ranked treatment among the many suggested interventions for pyoderma gangrenosum. ${ }^{7}$ None the less, previous studies have reported large proportions of people with pyoderma gangrenosum achieving complete response with ciclosporin, ${ }^{19-23}$ which led the current researchers of the STOP GAP (Study of Treatments fOr Pyoderma GAngrenosum Patients) randomised controlled trial to test the hypothesis that ciclosporin was superior to prednisolone for the treatment of pyoderma gangrenosum.

Healing responses at six weeks in our study were broadly similar to those observed for the randomised controlled trial of infliximab compared with placebo. ${ }^{9}$ In the STOP GAP trial, ulcers had healed at six weeks in 15\% of participants in the ciclosporin group and $21 \%$ in the prednisolone group. By comparison, ulcers had healed at six weeks in $21 \%$ of participants in the infliximab trial (all participants who had not responded to treatment at week 2 were offered infliximab regardless of the randomised allocation to infliximab or placebo). ${ }^{9}$ Subsequent observational studies suggest that treatment with antitumour necrosis factor is potentially more effective in patients with inflammatory bowel disease, ${ }^{24} 25$ but we did not have the power to look at this in the current trial. Head to head comparisons of antitumour necrosis factor with ciclosporin or prednisolone are needed, along with investigation of topical interventions that may provide a better risk-benefit profile for patients.

This trial is four times larger than the only other randomised controlled trial conducted in pyoderma gangrenosum, and it required national collaboration

Table 5 | Characteristics of changes in target lesions (erythema, border elevation, and exudate) as assessed by investigator at final visit

\begin{tabular}{|c|c|c|}
\hline $\begin{array}{l}\text { Assessment of } \\
\text { target lesions }\end{array}$ & $\begin{array}{l}\text { No }(\%) \text { in } \\
\text { ciclosporin group } \\
(n=57)\end{array}$ & $\begin{array}{l}\text { No }(\%) \text { in } \\
\text { prednisolone group } \\
(n=51)\end{array}$ \\
\hline \multicolumn{3}{|l|}{ Erythema: } \\
\hline Worse & $6(11)$ & $3(6)$ \\
\hline Same & $11(19)$ & $10(20)$ \\
\hline Improved & $40(70)$ & $38(75)$ \\
\hline \multicolumn{3}{|l|}{ Border elevation: } \\
\hline Worse & $2(4)$ & $8(16)$ \\
\hline Same & $15(26)$ & $9(18)$ \\
\hline Improved & $40(70)$ & $34(67)$ \\
\hline \multicolumn{3}{|l|}{ Exudate: } \\
\hline Worse & $5(9)$ & $4(8)$ \\
\hline Same & $7(12)$ & $8(16)$ \\
\hline Improved & $45(79)$ & $39(76)$ \\
\hline
\end{tabular}




\begin{tabular}{|c|c|c|c|}
\hline \multirow[b]{2}{*}{ Secondary outcomes } & \multicolumn{2}{|c|}{ No in group, mean (SD) } & \multirow{2}{*}{$\begin{array}{l}\text { Mean difference (ciclosporin-prednisolone) } \\
\text { adjusted mean difference* }(95 \% \mathrm{CI}) \text {, P value }\end{array}$} \\
\hline & Ciclosporin group & Prednisolone group & \\
\hline \multicolumn{4}{|l|}{ Pain scores (range 0-4): } \\
\hline Week 1 & $n=47,1.98(1.0)$ & $\mathrm{n}=38,1.84(1.2)$ & \\
\hline Week 2 & $\mathrm{n}=46,1.74(1.1)$ & $n=37,1.69(1.3)$ & \\
\hline Week 3 & $n=46,1.59(1.0)$ & $\mathrm{n}=36,1.48(1.2)$ & \\
\hline Week 4 & $\mathrm{n}=45,1.34(1.2)$ & $\mathrm{n}=35,1.50(1.2)$ & \\
\hline Week 5 & $\mathrm{n}=46,1.22(1.1)$ & $\mathrm{n}=34,1.49(1.3)$ & \\
\hline Week 6 & $\mathrm{n}=45,1.10(1.0)$ & $\mathrm{n}=32,1.49(1.3)$ & \\
\hline Weeks 1-6: AUC (0-20) & $n=45,7.5(4.8)$ & $n=32,7.9(5.6)$ & $-0.40-0.48(-2.82$ to 1.87$), 0.69$ \\
\hline \multicolumn{4}{|c|}{ DLQI (range 0-30, high score=worse): } \\
\hline Baseline & $\mathrm{n}=58,10.3(7.3)$ & $\mathrm{n}=53,13.2(9.0)$ & \multirow{2}{*}{$-1.5,-0.45$ ( -3.46 to 2.56$), 0.77$} \\
\hline Final visit & $\mathrm{n}=38,4.8(6.8)$ & $\mathrm{n}=28,6.3(7.6)$ & \\
\hline \multicolumn{4}{|c|}{ EQ-5D-3L (range-0.594-1.000, (low scores=worse): } \\
\hline Baseline & $n=56,0.51(0.35)$ & $\mathrm{n}=52,0.44(0.38)$ & \multirow{2}{*}{$0.13,0.13(-0.02$ to 0.28$), 0.095$} \\
\hline Final visit & $n=42,0.76(0.30)$ & $\mathrm{n}=27,0.63(0.41)$ & \\
\hline \multicolumn{4}{|c|}{ EQ VAS (range 0-100, low scores=worse): } \\
\hline Baseline & $n=57,62.6(22.2)$ & $n=53,61.4(21.5)$ & \multirow{2}{*}{$2.6,0.48$ (-9.3 to 10.3), 0.92} \\
\hline Final visit & $\mathrm{n}=41,73.2(20.5)$ & $n=29,70.6(22.3)$ & \\
\hline
\end{tabular}

$\mathrm{AUC}=$ area under the curve; $\mathrm{DLQI}=$ dermatology life quality index; EQ-5D-3L=European quality of life-5 dimensions, three levels; EQ VAS=European quality of life visual analogue scale.

${ }^{*}$ Adjusted for baseline values and stratification variables (lesion size and presence of underlying disease).

\section{Table 7 | Specific adverse reactions occurring in 3\% or more of participants in either} treatment group

\begin{tabular}{|c|c|c|}
\hline Upper and lower level classifications & $\begin{array}{l}\text { No }(\%) \text { in ciclosporin } \\
\text { group }(n=59)\end{array}$ & $\begin{array}{l}\text { No }(\%) \text { in prednisolone } \\
\text { group }(n=53)\end{array}$ \\
\hline \multicolumn{3}{|l|}{ Blood and the lymphatic system disorders: } \\
\hline Anaemia & $2(3)$ & $0(0)$ \\
\hline Leucocytosis & $0(0)$ & $5(9)$ \\
\hline \multicolumn{3}{|l|}{ Endocrine disorders: } \\
\hline Diabetes & $0(0)$ & $3(6)$ \\
\hline \multicolumn{3}{|l|}{ Metabolism and nutrition disorders: } \\
\hline Hyperglycaemia & $0(0)$ & $5(9)$ \\
\hline \multicolumn{3}{|l|}{ Nervous system disorders: } \\
\hline Tremor & $5(8)$ & $2(4)$ \\
\hline Headache & $5(8)$ & $0(0)$ \\
\hline Paraesthesia & $2(3)$ & $0(0)$ \\
\hline Euphoria & $0(0)$ & $3(6)$ \\
\hline Depression & $1(2)$ & $2(4)$ \\
\hline \multicolumn{3}{|l|}{ Gastrointestinal disorders: } \\
\hline Nausea & $12(20)$ & $1(2)$ \\
\hline Vomiting & $4(7)$ & $0(0)$ \\
\hline Diarrhoea & $2(3)$ & $0(0)$ \\
\hline Candidiasis & $1(2)$ & $2(4)$ \\
\hline \multicolumn{3}{|l|}{ Cardiovascular disorders: } \\
\hline Hypertension & $10(17)$ & $4(7)$ \\
\hline Oedema & $0(0)$ & $2(4)$ \\
\hline \multicolumn{3}{|l|}{ Hepatobiliary disorders: } \\
\hline Hepatic dysfunction & $2(3)$ & $1(2)$ \\
\hline \multicolumn{3}{|l|}{ Skin and subcutaneous tissue disorders: } \\
\hline Hypertrichosis & $2(3)$ & $0(0)$ \\
\hline \multicolumn{3}{|c|}{$\begin{array}{l}\text { Musculoskeletal, connective tissue and bone } \\
\text { disorders: }\end{array}$} \\
\hline Muscle cramps & $2(3)$ & $0(0)$ \\
\hline Myalgia & $2(3)$ & $1(2)$ \\
\hline Arthralgia & $2(3)$ & $0(0)$ \\
\hline \multicolumn{3}{|l|}{ Renal and urinary disorders: } \\
\hline Renal dysfunction & $18(30)$ & $1(2)$ \\
\hline \multicolumn{3}{|l|}{ General disorders: } \\
\hline Serious infection*: & $0(0)$ & $6(11)$ \\
\hline Other infection & $4(7)$ & $5(9)$ \\
\hline Fatigue & $2(3)$ & $4(7)$ \\
\hline Weight increase & $1(2)$ & $4(7)$ \\
\hline
\end{tabular}

One suspected unexpected serious adverse reaction (a ruptured abdominal aortic aneurysm) was reported during the trial.

${ }^{*}$ Requiring hospital admission or parenteral antibiotics. through the UK Dermatology Clinical Trials Network. ${ }^{26}$ Patient recruitment from 39 UK hospitals ensured representative sampling; the protocol reflected normal clinical practice, with dosing adjusted according to clinical need; and outcomes included clinician assessed, patient assessed, and independent assessment of digital images.

Every effort was made to capture the primary outcome in a blinded fashion, and all secondary analyses were supportive of this main analysis, although power to explore the impact on quality of life was limited owing to missing data from postal questionnaires.

Given the lack of a placebo or no treatment third arm in this study, it is possible that neither drug is effective in treating pyoderma gangrenosum. However, it was considered unethical to leave patients with a serious, potentially fatal disease, without treatment.

The obtained sample size was slightly smaller than had been planned, but the narrow confidence intervals for between group comparisons suggest that we did not miss clinically important differences. The trial was stopped after achieving $86 \%$ of target recruitment owing to time and financial limitations. This decision was made before the database was locked and data analysed.

\section{Conclusion}

Patients who require systemic treatment are likely to respond similarly to prednisolone or ciclosporin in the short term, but neither treatment is especially effective when healing at six months is considered. Differences in side effect profiles should be taken into account when choosing treatments. Table 8 provides an outline of key comorbidities that clinicians may wish to consider when choosing between prednisolone and ciclosporin for individual patients.

These results provide robust evidence to inform shared treatment decision making between clinicians and patients, including information on duration of treatment, response rates, adverse effect profiles, and likelihood of 


$\begin{aligned} & \text { Table } \mathbf{8} \mid \text { Authors' suggestions on when to consider prednisolone versus ciclosporin for } \\
& \text { patients with pyoderma gangrenosum based on pre-existing comorbidities }\end{aligned}$
\begin{tabular}{lll} 
Comorbidities & Favours prednisolone & Favours ciclosporin \\
Obesity $(>100 \mathrm{~kg})$ & Some concerns & Yes \\
\hline Diabetes & Strong reason for not using & Yes \\
\hline Hypertension & Some concerns & Strong reason for not using \\
\hline Renal insufficiency & Yes & Strong reason for not using \\
\hline Osteoporosis & Strong reason for not using & Yes \\
\hline Peptic ulceration & Strong reason for not using & Yes \\
\hline Malignancy & Yes & Some concerns \\
\hline History of mental illness & Some concerns & Yes
\end{tabular}

recurrence. However, our results suggest that better treatments for pyoderma gangrenosum are urgently needed. This trial was made possible by the collaborative efforts of doctors, nurses, and administrators at the recruiting hospitals. We thank everyone who contributed their time and expertise, in particular the trial participants and those who contributed to feasibility and pilot work for the subsequent trial. Their input and understanding were important in ensuring the success of this important trial. This work is subject to Crown Copyright.

Contributors: ADO and KST are joint first authors and act as guarantors. The STOP GAP trial was supported by research nurses and administrators provided through the National Institute for Health Research Clinical Research Networks in England, CRC Cymru Research Network in Wales, and NHS Research Scotland for Scotland. We also thank Bryony Elliot, Joanne Perdue, and Suyin Ong for their assistance with the assessment of outcomes from the digital images. In addition to the writing team, Becky Fox Spencer (independent medical writer) and Natasha Rogers (authors' editor and scientific writer) helped to draft the final manuscript.

Funding: This publication presents independent research funded by the National Institute for Health Research (NIHR) under its programme grants for applied research (RP-PG-0407-10177). The views expressed in this publication are those of the authors and not necessarily those of the NHS, the NIHR, or the Department of Health. Support for this trial was provided through Nottingham Clinical Trials Unit, the UK Dermatology Clinical Trials Network, the Robertson Centre for Biostatistics, and the Centre for Healthcare Randomised Trials. There was no commercial support for this study. The writing team all had input into the design and conduct of the trial, writing of the paper and/or performed the statistical analysis. All the authors vouch for the completeness and accuracy of the data presented and for the fidelity of the study to the protocol.

Competing interests: All authors have completed the ICMJE uniform disclosure form at www.icmje.org/coi disclosure.pdf and declare: no support from any organisation for the submitted work; no financial relationships with any organisations that might have an interest in the submitted work in the previous three years; no other relationships or activities that could appear to have influenced the submitted work.

Ethical approval: This study was approved by the National Research Ethics Service North East-York Committee (09/H0903/5), the Medicines and Healthcare Products Regulatory Agency (19162/0213/001), and European Clinical Trials database EudraCT (2008-008291-14).

Data sharing: Patient level data, the full dataset and statistical code are available from the corresponding author (kim.thomas@ nottingham.ac.uk). Participant consent for data sharing was not obtained but the presented data are anonymised and risk of identification is low.

Transparency: The lead authors (ADO and KST) affirm that the manuscript is an honest, accurate, and transparent account of the study being reported; that no important aspects of the study have been omitted; and that any discrepancies from the study as planned (and, if relevant, registered) have been explained.

This is an Open Access article distributed in accordance with the terms of the Creative Commons Attribution (CC BY 4.0) license, which permits others to distribute, remix, adapt and build upon this work, for commercial use, provided the original work is properly cited. See: http:// creativecommons.org/licenses/by/4.0/.
1 Langan SM, Groves RW, Card TR, et al. Incidence, mortality, and disease associations of pyoderma gangrenosum in the United Kingdom: a retrospective cohort study. I Invest Dermatol 2012;132:2166-70.

2 Brooklyn T, Dunnill G, Probert C. Diagnosis and treatment of pyoderma gangrenosum. BMJ 2006;333:181-4.

3 Binus AM, Qureshi AA, Li VW, et al. Pyoderma gangrenosum: a retrospective review of patient characteristics, comorbidities and therapy in 103 patients. Br J Dermatol 2011;165:1244-50.

4 Su WP, Davis MD, Weenig RH, et al. Pyoderma gangrenosum: clinicopathologic correlation and proposed diagnostic criteria. Int J Dermatol 2004;43:790-800

5 Tremezaygues L, Schmaltz R, Vogt T, et al. [Management of pyoderma gangrenosum. An update on clinical features, diagnosis and therapy] Hautarzt 2010;61:345-53; quiz 54-5.

6 Zuo KJ, Fung E, Tredget EE, et al. A systematic review of post-surgical pyoderma gangrenosum: Identification of risk factors and proposed management strategy. J Plast Reconstr Aesthet Surg 2015;68:295-303.

7 Miller J, Yentzer BA, Clark A, et al. Pyoderma gangrenosum: a review and update on new therapies. I Am Acad Dermatol 2010;62:646-54.

8 British Association of Dermatologists. Patient Information Leaflet on pyoderma gangrenosum. 2013. www.bad.org.uk/for-the-public/ patient-information-leaflets/pyoderma-gangrenosum.

9 Brooklyn TN, Dunnill MG, Shetty A, et al. Infliximab for the treatment of pyoderma gangrenosum: a randomised, double blind, placebo controlled trial. Gut 2006;55:505-9.

10 Reichrath J, Bens G, Bonowitz A, et al. Treatment recommendations for pyoderma gangrenosum: an evidence-based review of the literature based on more than 350 patients. J Am Acad Dermatol 2005;53:273-83.

11 Craig FF, Thomas KS, Mitchell EJ, et al. UK Dermatology Clinical Trials Network's STOP GAP trial (a multicentre trial of prednisolone versus ciclosporin for pyoderma gangrenosum): protocol for a randomised controlled trial. Trials 2012;13:51.

12 RALLOC: Stata module to design randomized controlled trials [program]. Boston College Department of Economics, 1997.

13 Kantor J, Margolis DJ. A multicentre study of percentage change in venous leg ulcer area as a prognostic index of healing at 24 weeks. BrJ Dermatol 2000;142:960-4

14 Cardinal M, Phillips T, Eisenbud DE, et al. Nonlinear modeling of venous leg ulcer healing rates. BMC Dermatol 2009;9:2

15 Foss CE, Clark AR, Inabinet R, et al. An open-label pilot study of alefacept for the treatment of pyoderma gangrenosum. J Eur Acad Dermatol Venereol 2008;22:943-9.

16 Finlay AY, Khan GK. Dermatology Life Quality Index (DLQI) - a simple practical measure for routine clinical use. Clin Exp Dermatol 1994;19:210-6.

17 Kind P, Dolan P, Gudex C, et al. Variations in population health status: results from a United Kingdom national questionnaire survey. $B M$ J 1998;316:736-41.

18 Brooks R. EuroQol-the current state of play. Health Policy 1996;37:53-72

19 Matis WL, Ellis CN, Griffiths CE, et al. Treatment of pyoderma gangrenosum with cyclosporine. Arch Dermatol 1992;128:1060-4.

20 Elgart G, Stover P, Larson K, et al. Treatment of pyoderma gangrenosum with cyclosporine: results in seven patients. J Am Acad Dermatol 1991;24:83-6.

21 Vidal D, Puig L, Gilaberte M, et al. Review of 26 cases of classical pyoderma gangrenosum: clinical and therapeutic features. J Dermatolog Treat 2004;15:146-52.

22 Capella GL, Frigerio E, Fracchiolla C, et al. The simultaneous treatment of inflammatory bowel diseases and associated pyoderma gangrenosum with oral cyclosporin A. Scand J Gastroenterol 1999;34:220-1.

23 Friedman S, Marion JF, Scherl E, et al. Intravenous cyclosporine in refractory pyoderma gangrenosum complicating inflammatory bowel disease Inflamm Bowel Dis 2001:7:1-7.

24 Agarwal A, Andrews JM. Systematic review: IBD-associated pyoderma gangrenosum in the biologic era, the response to therapy. Aliment Pharmacol Ther 2013;38:563-72

25 Arguelles-Arias F, Castro-Laria L, Lobaton T, et al. Characteristics and treatment of pyoderma gangrenosum in inflammatory bowel disease. Dig Dis Sci 2013;58:2949-54.

26 Layfield C, Yong A, Thomas KS, et al. The UK Dermatology Clinical Trials Network-how far have we come? Clin Investig 2014;4:209-14.

(c) BMJ Publishing Group Ltd 2015

Supplementary appendix: members of STOP GAP trial team

Supplementary figure: measurement of pyoderma gangrenosum ulcer using image analysis software 